\title{
Early expression of axon guidance molecules in the embryonic chick mesencephalon and pretectum
}

\author{
KERRY-LYN RILEY\#, SARAH GLEDHILL and FRANK R. SCHUBERT* \\ Institute of Biomedical and Biomolecular Sciences, University of Portsmouth, U.K.
}

\begin{abstract}
Early axon tracts in the developing vertebrate brain are established along precise paths. Yet, little is known about axon guidance processes at early stages of rostral brain development. Using whole mount in situ hybridisation in combination with immunohistochemistry, we have analysed the expression patterns of Slits, Netrins, Semaphorins and the respective receptors during the formation of the early axon scaffold, particularly focusing on the pretectalmesencephalic boundary. Many of these guidance molecules are expressed in close correlation with the growing tracts, and the nuclei of the corresponding neurons often express the respective receptors. The expression patterns of Slits and Netrins implicate them with the positioning of the longitudinal tracts along the dorsoventral axis, while Semaphorins could provide guidance at specific choice points. Our study provides a catalogue of gene expression for future studies on axon guidance mechanisms in the early brain.
\end{abstract}

KEY WORDS: early axon scaffold, netrin, slit, semaphorin

\section{Introduction}

The first differentiating neurons in the embryonic vertebrate brain form an early scaffold of axon tracts (Chedotal et al., 1995; Chitnis and Kuwada, 1990; Doldan et al., 2000; Easter et al., 1993; Hartenstein, 1993; Ishikawa et al., 2004; Mastick and Easter, 1996; reviewed in Nieuwenhuys, 1998; Wilson et al., 1990). The early axon scaffold is made up of several longitudinal, transversal and commissural tracts including the medial longitudinal fascicle (MLF) and the posterior commissure (PC), both originating from the midbrain-forebrain border (MFB) region (reviewed in Ahsan et al., 2007). It has been hypothesised that the early axon scaffold sets up major pathways in the brain by forming substrates for future follower axons (Chitnis and Kuwada, 1991, reviewed in Hjorth and Key, 2002). Disruptions in the formation of these early tracts would therefore lead to the miswiring of tracts developing later on. In line with this idea, removing early neurons in the ventral MFB region results in later PC axons extending along aberrant pathways (Chitnis and Kuwada, 1991; Patel et al., 1994). Likewise, the early MLF axons have been implicated with the formation of the reticulospinal projection in the hindbrain (Hernandez-Montiel et al., 2003). Within the chick brain, several other tracts develop early that have not been considered in the descriptions of the early axon scaffold development in anamniotes (Fig. 1). In particular, neurons of the mesencephalic nucleus of the trigeminal nerve and the tectobulbar tract contribute axons to the lateral longitudinal fascicle (LLF) that is evident in the chick brain from Hamburger \& Hamilton $(\mathrm{HH})$ stage 17. Motorneurons in the oculomotor nucleus are also present early in the mesencephalon of chick embryos.

The axons of the early axon scaffold follow precise paths, suggesting that their projection is controlled by guidance cues. Most long-range axon guidance signalling is attributed to members of just three, highly conserved families of secreted guidance molecules, Netrins, Slits, and Class3 Semaphorins (reviewed in Chilton, 2006; Cooper, 2002; Dickson, 2002). Vertebrate Netrins were initially isolated as floor plate-derived factors that promote the extension of commissural axons in the spinal cord (Serafini et al., 1994). Subsequent studies showed that they can act as either a chemorepellent or chemoattractant, depending on the receptors present on the developing axons (reviewed in Moore et al.,

Abbreviations used in this paper: E, embryonic day (of incubation); HH, Hamburger \& Hamilton stage; LLF, lateral longitudinal fascicle; MFB, midbrain-forebrain border; MHB, midbrain-hindbrain border; MLF, medial longitudinal fascicle; $\mathrm{nMLF}$, nucleus of the MLF; PC, posterior commissure.

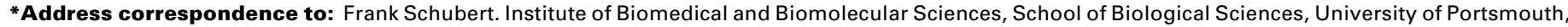
King Henry Building, King Henry I Street, Portsmouth PO1 2DY, United Kingdom. Fax: +44-(0)23-9284-2070. e-mail: Frank.Schubert@port.ac.uk web: http://www.port.ac.uk/research/ibbs/genesanddevelopment/frankschubertslab/

\# Current address: MRC Mammalian Genetics Unit, Harwell, U.K.
} 
2007; Tessier-Lavigne and Goodman, 1996). Binding of Netrin to DCC (Keino-Masu et al., 1996) or its paralogue, Neogenin (Vielmetter et al., 1994), mediates a chemoattractive response, while binding to Unc5 (Leonardo et al., 1997) alone or in combination with Neogenin leads to a repulsion of developing axons. Interestingly, DCC has not been described in the chick, despite the conservation in other vertebrates. Slits are chemorepulsive axon guidance molecules, best known for their role in preventing the re-crossing of commissural axons at the midline of Drosophila (Tear et al., 1993). In vertebrates, three Slit homologues and two homologues of the Robo receptor have been identified (Brose et al., 1999; Vargesson et al., 2001). Along with the divergent receptor Rig-1, they are involved in controlling commissure formation in the spinal cord (reviewed in Dickson and Gilestro, 2006). The secreted Class 3 Semaphorins, initially termed "Collapsins", were first identified due to their ability to collapse growth cones (Luo et al., 1993; Luo et al., 1995). They form a large family of guidance molecules with seven members identified in amniotes alone, but appear restricted to vertebrates (reviewed in Raper, 2000). The Class3 Semaphorins bind to the Neuropilin receptors (He and Tessier-Lavigne, 1997; Kolodkin et al., 1997), which lack an intracellular domain and therefore have to form a complex with the Plexin co-receptors to mediate signal transduction (Takahashi et al., 1999; Tamagnone et al., 1999). Two Neuropilins and several Plexins have been found in vertebrates (reviewed in Raper, 2000).

While the course of the early tracts in the embryonic vertebrate brain has been documented in several species including the chick (Chedotal et al., 1995), the molecular mechanisms governing axon guidance along individual pathways of the early axon scaffold are less well understood. Previous investigations in Xenopus have implicated Netrin and Semaphorin signalling in the formation of the ventral commissure (Anderson et al., 2000a; Anderson et al., $2000 \mathrm{~b}$ ), while Slits are involved in the fasciculation of the MLF in chick (Molle et al., 2004) and formation of the tract of the postoptic commissure in zebrafish (Devine and Key, 2008). Yet, generally little is known about the expression of guidance molecules in the rostral brain in relation to the developing tracts. A study in zebrafish highlighted the correlation of gene expression patterns with axon tracts (Hjorth and Key, 2001), but investigated patterning genes rather than axon guidance molecules. The aim of this study was to analyse the expression of the major groups of axon guidance molecules within ventral pretectum and mesencephalon, and to correlate their expression pattern with the position of neurons and axon tracts. Our results show that Netrins, Slits and Semaphorins and their receptors are already expressed during the initial outgrowth of the first axons in the rostral brain and indicate possible guidance mechanisms for the longitudinal tracts.

\section{Results}

To investigate the expression of axon guidance molecules within the ventral mesencephalon and pretectum, the temporal and spatial expression of several families of axon guidance molecules, including Slits, Netrins and
Class3 Semaphorins, were visualised using in situ hybridisation on embryos incubated for 2 days (E2, HH13-15), 3 days (E3, $\mathrm{HH} 17-18)$ and 4 days (E4, $\mathrm{HH} 20-21)$, i.e. between the start of axon tract formation in the brain and the appearance of the fully formed early axon scaffold. In situ hybridisation was combined with immunohistochemistry, using an anti-neurofilament antibody, to allow simultaneous visualisation of guidance cue expression and axon tracts.

\section{Netrins and their receptors}

The best-characterised vertebrate Netrin receptor is DCC (Keino-Masu et al., 1996), which however has not yet been reported in chick. When our attempts to isolate chick $D C C$ by PCR with degenerate primers failed, we queried the chick genome sequence for DCC and found evidence for a deletion of the whole $D C C$ locus during bird evolution (manuscript in preparation). Hence, with $D C C$ apparently missing in chick, Netrin binding relies largely on Neogenin and the second receptor class, Unc5, although recent studies suggest that other proteins like Dscam can also act as Netrin receptors (Andrews et al., 2008). We have analysed the expression patterns of the axon guidance mol-

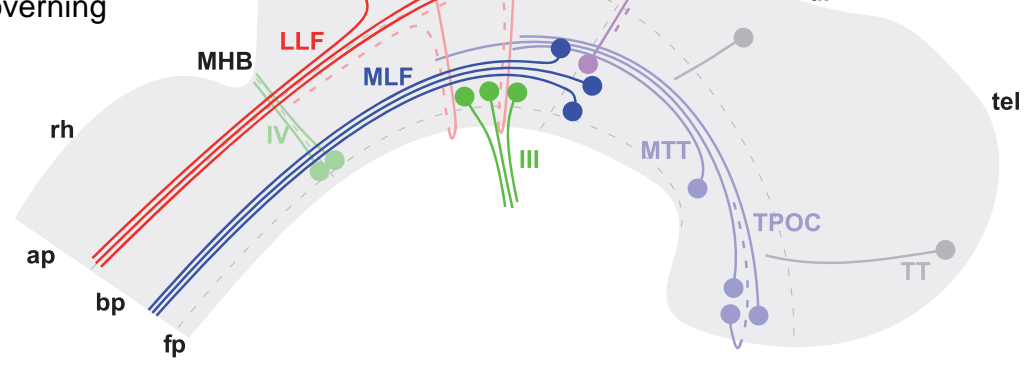

Fig. 1. Schematic representation of early brain organisation in the chick. The diagram depicts regions, neuron clusters and axon tracts in the early embryonic chick brain at E4. The prominent ventral longitudinal bundle (blue) is made up of the TPOC originating in the hypothalamus, the MTT from the ventral diencephalon, and the MLF from the ventral midbrain-forebrain border. At this stage, all axons in the ventral bundle project caudally. The LLF in the mesencephalon is formed of ipsilateral axons from the DTmesV and contralateral axons from the TB (red). The DTmesV neurons are located along the dorsal midline of the mesencephalon and project their axons ventrally along the tectum before turning sharply caudally. The TB fibres extend from the tectum ventrally across the ventral midline before joining the contralateral LLF. Just rostral to the MFB, transversal axons form the TPC (pink). Neurons contributing to the TPC are located dorsally and ventrally in the caudal pretectum. Somatic motor neurons at the $M H B$ (trochlear) and in the mesencephalon (ocular) yield two of the cranial nerves (green). The tracts that are the focus of this study (MLF, DTmesV, III) are shown in darker colour. Key: III (oculomotor nerve), IV (trochlear nerve), ap (alar plate), bp (basal plate), di (diencephalon), DTmesV (dorsal tract of the mesencephalic nucleus of the trigeminal nerve), fp (floor plate), LLF (lateral longitudinal fascicle), MFB (midbrainforebrain border), MHB (midbrain-hindbrain border), MLF (medial longitudinal fascicle), mes (mesencephalon), MTT (mamillotegmental tract), pt (pretectum), rh (rhombencephalon), TB (tectobulbar tract), tel (telencephalon), TPC (tract of the posterior commissure), TPOC (tract of the postoptic commissure), TT (telencephalic tract). 
Fig. 2. Expression of Netrins and their receptors. Embryos at stages E2, E3 and E4 were analysed, using in situ hybridisation for the expression of Netrin1, Netrin2, Unc5H3, and Neogenin (blue staining, black arrowheads) within the ventral mesencephalon. Neurons (brown) were visualised using an antineurofilament antibody. In photographs of E2 embryos, rostral is orientated to the right and the ventral midline (line) runs through the middle of the photograph. The images of later stages focus on the ventral midbrain, with rostral to the right and dorsal orientated towards the top. The positions of the midbrainhindbrain (MHB) and midbrain-forebrain borders (MFB) are indicated by lines. The scale bar is $100 \mu \mathrm{m}$. (A-C) Throughout the developmental stages investigated, Netrin 1 is expressed from the ventral floor plate up to and including the MLF. Weaker signal appears towards the more dorsal regions of this expression domain, particularly overlapping with MLF axons. (D-F) Netrin2 expression. (D) At HH14, Netrin2 is expressed in two areas, a small domain in the rostral mesencephalic basal plate, dorsal to the $n M L F$, and the basal plate of the MHB. (E) In HH18 embryos, Netrin2 expression is seen both rostral and caudal to the midbrain-forebrain boundary, bordering the MFB. The main midbrain expression domain is located in the rostral part of the ventral alar plate, ventrally delimited by the LLF. Further expression is restricted to the basal plate at the caudal most region of the mesencephalon and MHB. (F) At HH2O, expression of Netrin2 extends throughout the rostral tegmentum and pretectal region. Stronger expression spans from the floor plate across the MLF tract, whilst weaker expression is observed within the basal plate region of the rostral mesencephalon. (G-I) Unc5H3 expression. (G) At HH15, no Unc5H3 expression is observed. (H,I) At HH18 and HH21, Unc5H3 expression overlaps with the nMLF. (J-L) Neogenin expression. (J) At HH13, Neogenin is weakly expressed throughout the mesencephalon. (K) Neogenin expression continues in the basal plate at HH17, but is reduced in the alar plate of the midbrain. (L) By HH21, Neogenin signals are restricted to the mesencephalic alar plate. Abbreviations: LLF (lateral longitudinal fascicle), MFB (midbrain-forebrain border), MHB (midbrain-hindbrain border), MLF (medial longitudinal fascicle), mes (mesencephalon), nIIl (oculomotor nucleus), pt (pretectum), rh (rhombencephalon).

ecules, Netrin1 and Netrin2, and the receptors, Unc5H3 and Neogenin (Fig. 2) in the ventral mesencephalon. The two Netrin ligands display distinct expression patterns in the midbrain. Throughout the developmental stages investigated, Netrin 1 was expressed as a gradient reaching from the floor plate into the ventral midbrain (Fig. $2 \mathrm{~A}-\mathrm{C}$ ). The Netrin 1 signals in the mesencephalon were confined to floor plate and ventral basal plate, covering the MLF. The expression pattern of Netrin2 is more complex and dynamic. At HH14, Netrin2 is expressed in a distinct pattern within the midbrain (Fig. 2D). Caudally, Netrin2 signals were observed at the ventral midbrain-hindbrain boundary (MHB) and the hindbrain. The rostral expression domain is located dorsal to the nucleus of the MLF (nMLF) in the rostral mesencephalon. At HH18, Netrin2is expressed in the rostral half of the mesencephalon dorsal to and overlapping the LLF, and also in a patch abutting the MFB between LLF and oculomotor nucleus (Fig. 2E). In addition, Netrin2 signals were apparent caudally around the $\mathrm{MHB}$, and rostrally in the pretectum, rostral to the commissural region. By HH2O, Netrin2 is expressed throughout the whole ventral mesencephalon, at high levels in the floor plate, and at lower level between floor plate and LLF (Fig. 2F).

Unc5H3 expression was not detectable at $\mathrm{HH} 15$ (Fig. 2G). At $\mathrm{HH} 18$, Unc5H3 is expressed by individual neurons at the MFB, indicated by the speckled pattern of expression (Fig. 2H), while the Unc5H3 staining broadly covered the area of the nMLF at $\mathrm{HH} 21$ (Fig. 2l). Neogenin at HH13 appeared to be expressed ubiquitiously at very low levels (Fig. 2J). At HH17, Neogenin signals were stronger in the basal plate of the mesencephalon, possibly even absent from the alar plate (Fig. 2K), while at HH21 the staining became restricted to the alar plate, from the LLF up to the roof plate (Fig. 2L).

\section{Slits and Robo receptors}

We have analysed the expression of Slit1, Slit2 and Slit3, and 


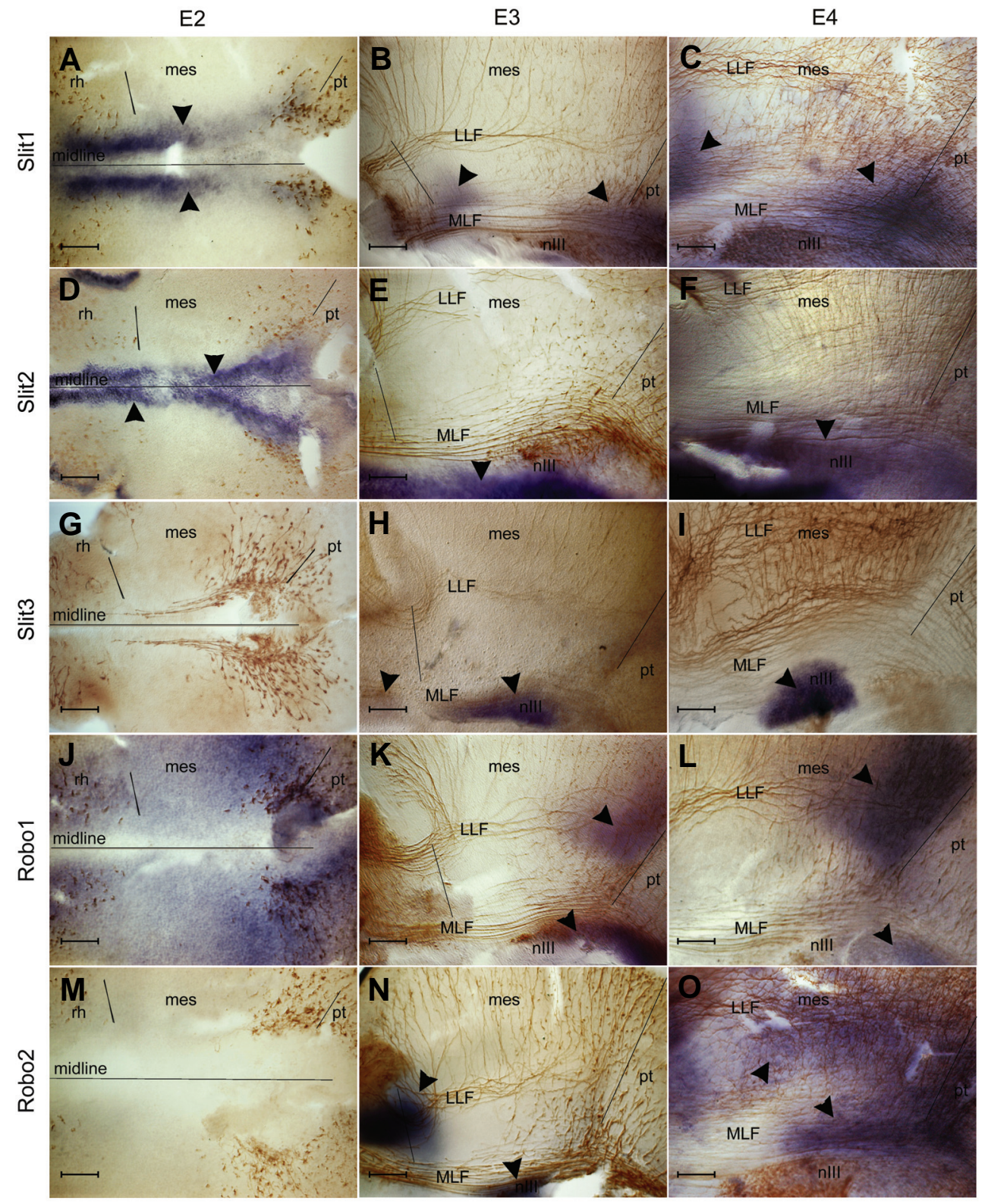

Fig. 3. Expression of Slits and their receptors. The expression of Slit1, Slit2, Slit3, Robo1 and Robo2 was visualised using RNA in situ hybridisation (blue staining, black arrowheads). The neurons have been stained using immunohistochemistry with an anti-neurofilament antibody. In photographs of E2 embryos, rostral is orientated to the right and the ventral midline (line) runs through the middle of the photograph. The images of later stages focus on the ventral midbrain, with rostral to the right and dorsal orientated towards the top. The positions of MHB and MFB are indicated by lines. The scale bar is $100 \mu \mathrm{m}$. (A-C) Slit1 expression. (A) At HH13, Slit1 is expressed in a narrow stripe of the mesencephalic, ventral basal plate, running along the ventral midline. (B,C) At later stages, Slit1 expression is restricted to two domains, one broadly overlying the $n M L F$ and the second at the ventral MHB. (D-F) Slit2 expression is seen throughout the ventral floor plate, extending up to but not overlapping the MLF. (G-I) Slit3 expression. (G) At HH14, no Slit3 signals are detectable in the brain. $(\mathbf{H}, \mathbf{I})$ At later stages, Slit3 is expressed in the oculomotor nucleus. (J-L) Robo1 expression. (J) Weak Robo1 expression is visible throughout basal and alar plates at HH13, with stronger staining located in the rostral mesencephalon. $\mathbf{( K , L ) ~ R o b o 1 ~ e x p r e s s i o n ~ l a t e r ~}$ becomes restricted to the rostral mesencephalon; one domain is located within the tectal region of the mesencephalon whilst a second domain overlaps the $M M L F$. (M-O) Robo2 expression. (M) No Robo2 expression is seen at HH13. (N) At HH18, Robo2 expression overlies the oculomotor nucleus and is located within the basal plate region of the caudal mesencephaIon. (O) In the HH21 brain, the Robo2 expression domain has expanded in size, spanning the dorsal mesencephalon, and ventrally labelling the rostral mesencephalon including nMLF and oculomotor nucleus. Key: LLF (lateral longitudinal fascicle), MLF (medial longitudinal fascicle), mes (mesencephalon), nlll (oculomotor nucleus), pt (pretectum), rh (rhombencephalon).

the receptors, Robo1 and Robo2 (Fig. 3) in the embryonic chick midbrain. Slit1 and Slit2 ligands were found to be expressed around the ventral midline already at $\mathrm{HH} 13$. At this early stage, Slit1 is expressed in a narrow domain adjacent to the floor plate throughout the whole mesencephalon, extending into the hindbrain (Fig. 3A). Slit1 signals were stronger in the caudal mesencephalon, but also covered the nMLF rostrally. At HH18 and $\mathrm{HH} 21$, the expression is separated into two ventral expression domains, a rostral area broadly overlying the nMLF at the MFB, and a caudal area around the MHB (Fig. 3 B,C). While the rostral expression domain is confined to ventral aspects of the basal plate, the caudal domain extends dorsally from the MLF, reaching the LLF by HH21. In contrast to the dynamic Slit1expression, Slit2 expression between $\mathrm{HH}_{13}$ and $\mathrm{HH} 21$ is invariably limited to a ventral stripe (Fig. $3 \mathrm{D}-\mathrm{F}$ ). Starting at the floor plate, at later stages Slit2 signals later spread into the ventral basal plate, dorsally limited by the MLF. Slit3 expression was not detectable at the early stage (Fig. $3 \mathrm{G}$ ), but at $\mathrm{HH} 18$ is present in the oculomotor nucleus (Fig. $3 \mathrm{H}$ ). At $\mathrm{HH} 21$, Slit3 expression persists in the oculomotor nucleus (Fig. 3I), and could also be detected in the trochlear nucleus (not shown).

At $\mathrm{HH} 13$, Robo1 is expressed throughout the embryonic midbrain except the floor plate (Fig. 3J), with strongest expression rostrally around the MFB. At later stages, Robo1 expression becomes more confined. At HH18 and HH21, Robo 1 signals were observed in two distinct domains in the rostral mesencephalon (Fig. $3 \mathrm{~K}, \mathrm{~L}$ ): a ventral expression domain of Robo1 extends from the oculomotor nucleus up to and across the nMLF into the 


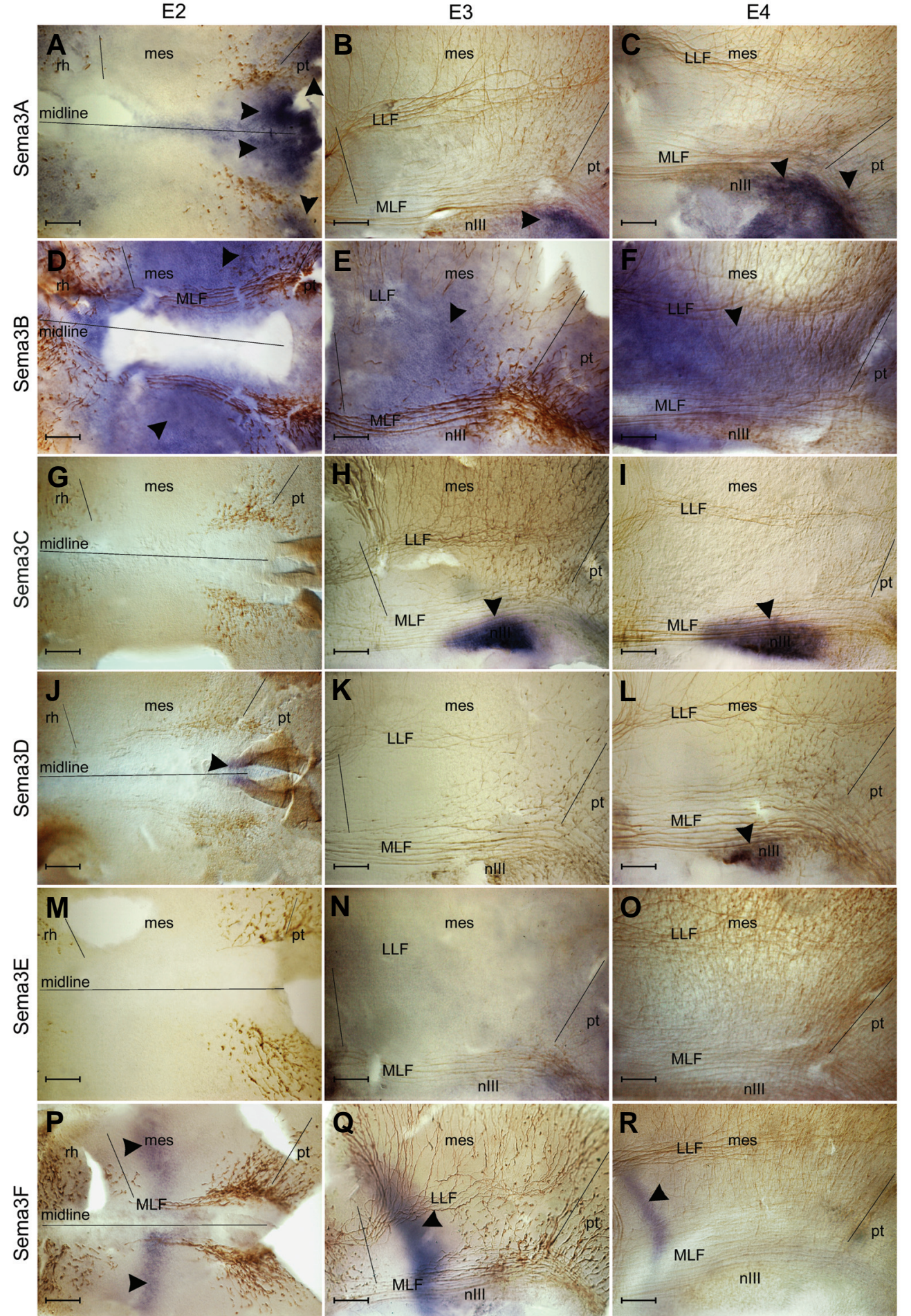

Fig. 4. Expression of Class 3 Semaphorins. The expression patterns of the Class3 Semaphorins in the early chick brain have been analysed using in RNA situ hybridisation (blue staining). This was coupled with immunohistochemistry using an anti-neurofilament antibody, allowing the visualisation of neurons (brown staining). In photographs of E2 embryos, rostral is orientated to the right and the ventral midline (line) runs through the middle of the photograph. The images of later stages focus on the ventral midbrain, with rostral to the right and dorsal orientated towards the top. The positions of MHB and MFB are indicated by lines. The scale bar is $100 \mu \mathrm{m}$. (A-C) Sema3A expression lies ventrally and rostrally to the $n M L F$ at all stages analysed. (A) At HH13, Sema3A is also observed in a domain lying within the alar plate of the rostral mesencephalon and dorsal pretectum. (D-F) Sema3B expression. (D) At HH14, Sema3B expression is observed in the basal and alar plates throughout the midbrain. (E,F) In the older stages, Sema3B expression is increasingly restricted to the basal plate of the mesencephalon. (G-I) Sema3C expression. (G) No Sema3C expression is observed at HH13. $(\mathbf{H}, \mathbf{I})$ In HH18 and HH2O embryos, Sema3C expression overlaps with the oculomotor nucleus. (J-L) Sema3D expression. (J) Early Sema3D expression occupies a small patch of floor plate in the pretectum. (K) At HH17, no Sema3D expression is seen within the mesencephalon. (L) From HH20, Sema3D expression overlaps with the caudal part of the oculomotor nucleus. (M-O) No Sema3E expression is observed in the embryonic chick mesencephalon. (P-R) In all the developmental stages investigated, the Sema3F expression domain forms a stripe in the caudal mesencephalon, close to the MHB. Key: LLF (lateral longitudinal fascicle), MLF (medial longitudinal fascicle), mes (mesencephalon), nIIl (oculomotor nucleus), pt (pretectum), rh (rhombencephalon). pretectum, while a dorsal domain spans the rostral mesencephaIon adjoining the MFB. In contrast to the early, broad expression of Robo1, no Robo2 signal was observed at HH13 (Fig. 3M). However, at $\mathrm{HH} 18$ Robo2 is expressed in two distinct domains, a caudal domain at the MHB between MLF and LLF, and a rostral domain overlying the oculomotor nucleus (Fig. 3N). At HH21, Robo2 signals were seen in a much larger area, covering the mesencephalon dorsal to the MLF. Rostrally, Robo2 is addition- ally expressed in a ventral area that covers a broad region around the $\mathrm{nMLF}$ (Fig. 30). The two expression domains appear to merge at the MFB.

\section{Class3 semaphorins and their receptors}

Previous studies have investigated Semaphorin expression in the hindbrain (Chilton and Guthrie, 2003) and the expression of selected Semaphorins and the Neuropilin receptors in the rostral 
Fig. 5. Expression of Class3 Semaphorin receptors. The expression of Neuropilins and Plexins (blue staining, black arrowheads) in the chick brain at E2, E3 and E4 was analysed in relation to the developing axon tracts (anti-neurofilament antibody, brown staining). In photographs of E2 embryos, rostral is orientated to the right and the ventral midline (line) runs through the middle of the photograph. The images of later stages focus on the ventral midbrain, with rostral to the right and dorsal orientated towards the top. The positions of MHB and MFB are indicated by lines. The scale bar is $100 \mu \mathrm{m}$. (A-C) Throughout the developmental stages analysed, Neuropilin 1 expression overlies the $n M L F$ and the caudal mesencephalon along the MHB. (D-F) Neuropilin2 is expressed in a domain spanning across the dorsal part of the $M H B$, extending into the rostral rhombencephalon and caudal mesencephalon. (E,F) From HH17 onwards, Neuropilin2 expression also overlaps with the oculomotor nucleus. (G-I) PlexinA1 expression. (G) PlexinA1 expression spans the entire length mesencephalon at HH13, ventrally delimited by the MLF. Expression levels in the caudal mesencephalon are lower than in the MHB and the rostral mesencephalon. (H,I) From HH17 onwards, PlexinA1 expression overlies the LLF but does not extend as far ventrally as the MLF. At HH21, PlexinA1 signals are additionally visible in the rostral, ventral basal plate of the mesencephalon (I). (JL) PlexinA4 expression overlaps with the nMLF (black arrowheads). Background staining throughout the mesencephalon partly obscures the signal, but the inset in (K) demonstrates PlexinA4 expression in individual MLF neurons at high magnification. (M-O) PlexinB1 expression. (M) At HH14, PlexinB1 is expressed widely in the mesencephalon exept the floor plate. (N) longitudinal fascicle), MLF (medial longitudinal fascicle), mes (mesencephalon), nIll (oculomotor nucleus), pt (pretectum), rh (rhombencephalon).

brain (Melendez-Herrera and Varela-Echavarria, 2006). We have characterised the expression of several Class 3 Semaphorins (Fig. 4) and the Neuropilin and Plexin receptors (Fig. 5) within the developing chick mesencephalon and pretectum in relation to the developing axon tracts.

The different Class3 Semaphorins showed distinct expression patterns during early brain development. Sema3A is expressed in a distinct domain in the ventral MFB and pretectum, located rostral and ventral to the nMLF (Fig. $4 \mathrm{~A}-\mathrm{C}$ ). At $\mathrm{HH} 13$, a second domain of Sema3A expression lies in the alar plate of the rostral mesencephalon and dorsal pretectum, dorsal to the MLF neurons (Fig. $4 \mathrm{~A})$. From HH18 onwards, this dorsal expression of Sema3A was no longer detectable, but Sema3Asignals were still observed in the MFB and pretectum, rostral and ventral to the nMLF (Fig. 4 B,C).
At stage $\mathrm{HH} 14$, Sema3Bis expressed throughout mesencephalon and pretectum apart from the floor plate (Fig. 4D). Sema3Bsignals in the alar plate are reduced by $\mathrm{HH} 17$ (Fig. 4E), and by HH21 the mesencephalic expression is restricted to the basal plate between LLF and floor plate (Fig. 4F). The expression of Sema3C is restricted to the oculomotor nucleus. While no staining was detectable at HH13 (Fig. 4G), strong Sema3C signals were seen at the older stages (Fig. $4 \mathrm{H}, \mathrm{I}$ ). Sema3D was found to be expressed in two different domains, depending on the developmental stage. At $\mathrm{HH} 14$, Sema3D is weakly expressed within the floor plate of the pretectum (Fig. 4J). At HH17, no Sema3D expression was seen throughout the mesencephalon and pretectum (Fig. 4K). At stage $\mathrm{HH} 20$, Sema3D signals overlay the caudal region of the oculomotor nucleus (Fig. 4L). No Sema3EmRNA could be detected within 
the mesencephalon, throughout the three developmental stages investigated (Fig. 4M-O). Sema3Fexpression at all stages analysed occupied a narrow region in the basal and alar plates of the caudal mesencephalon, aligning the MHB (Fig. 4 P-R).

At all stages analysed, Neuropilin1 was expressed in two distinct domains, a dorsoventral stripe in the caudal midbrain aligning the $\mathrm{MHB}$, and a smaller area at the ventral MFB, overlying the nMLF (Fig. $5 \mathrm{~A}-\mathrm{C}$ ). The expression pattern of Neuropilin2 is more dynamic. At HH14, Neuropilin2 is expressed in a dorsal domain in the caudal mesencephalon and rostral rhombencephaIon, extending across the isthmic region. This expression is stronger in the alar plate in both these regions (Fig. 5D). By HH17, this expression domain had broadened and extended ventrally, and a second Neuropilin2 domain overlapping the nucleus of the oculomotor nerve had appeared (Fig. 5 E-F). PlexinA1 was initially expressed widespread in the mesencephalon except the floor plate, though at lower levels along the MHB and in the rostral alar plate (Fig. 5G). From HH17 onwards the PlexinA1 signal focussed to the dorsal basal plate and ventral alar plate, around the LLF (Fig. $5 \mathrm{H})$. This domain extends across the pretectum, mesencephalon and rhombencephalon. By $\mathrm{HH} 21$, staining was also observed rostrally in a domain ventral to the MLF tract and rostral to the oculomotor nucleus (Fig. 5I). Similar to Neuropilin1, the expression pattern of PlexinA4 overlaps with the nMLF (Fig. $5 \mathrm{~J}-\mathrm{L}$ ). Barely visible at $\mathrm{HH} 13$, this staining became more distinct at older stages, and at $\mathrm{HH} 21$, strong PlexinA4 signals overlapped the nMLF (Fig. $5 \mathrm{~L})$. In contrast to the cell-specific expression of the other receptors, PlexinB1 was expressed throughout the neural tube except the floor plate at all stages analysed (Fig. $5 \mathrm{M}-\mathrm{O}$ ), although at later stages staining in the basal plate was much stronger than in the alar plate (Fig. $5 \mathrm{~N}$ ).

\section{Discussion}

Many of the early developing axon tracts in the vertebrate brain either originate in, or traverse through the midbrain and pretectum. Yet, little is known about the cues governing the outgrowth of these axons. We have investigated the expression patterns of the main secreted axon guidance molecules, Slits, Netrins and Class3 Semaphorins, and their receptors during early stages of brain development. Many of the guidance cues are expressed in distinct patterns during the growth of the pioneering axons, and several receptors correspondingly are expressed in specific groups of neurons. It is noteworthy that our analysis is limited to the mRNA level, and does not address the distribution of the secreted axon guidance proteins, or the actual presence of receptor molecules on the growth cones of the early axons.

\section{The longitudinal guidance system}

Two longitudinal axon tracts are formed in the early chick midbrain, the medial (MLF) and lateral (LLF) longitudinal fascicles. The MLF is formed by neurons located at the ventral MFB that project their axons caudally. The tract runs as a loose bundle adjacent to the floor plate through midbrain and hindbrain towards the spinal cord (Ahsan et al., 2007). The LLF is made up of two groups of neurons: neurons in the mesencephalic nucleus of the trigeminus are found dorsally, close to the roof plate, and send their axons ipsilaterally to the trigeminal ganglion; neurons dispersed in the tectum mostly project contralaterally, growing their axons across the midline before joining the LLF to form the tectobulbar tract.

The expression patterns of several axon guidance molecules are correlated with the longitudinal tracts. Notably, Netrins and Slits are expressed in distinct domains in or around the floor plate. In the spinal cord, these guidance cues control commissure formation, with Netrin acting as attractant and Slit as repellent (reviewed in Chilton, 2006; reviewed in Dickson and Gilestro, 2006). Similar to earlier reports describing Netrin 1 to be expressed by the floor plate up to the caudal diencephalon (Kennedy et al., 1994), we found strongest Netrin 1 expression in the floor plate of midbrain and pretectum, diminishing dorsally within the basal plate (Fig. 6 A,B). The expression domain includes the axons of the MLF, but the most intense Netrin 1 signal is located ventral to the MLF. In contrast to Netrin1, Netrin2expression is temporally and spatially associated with the appearance of the LLF, aligning the tract dorsally and ventrally (Fig. 6 A,B). Among the Netrin receptors, Neogenin is expressed broadly in the midbrain, while the expression domain of Unc5H3, the receptor mediating chemorepulsion by Netrins, correlates with the position of the MLF neurons (Fig. 6 C,D). We were unable to detect $D C C$ in chick, probably due to deletion of the gene, which is surprising since the knockout of $D C C$ in mice leads to neonatal death (Fazeli et al., 1997), and DCC and Neogenin have distinctly different expression patterns in mice (Gad et al., 1997). Slit expression at the isthmus has previously been shown to channel the MLF into a tight bundle (Molle et al., 2004). Similar to the study by Molle et al., we found Slit2 to be expressed ventral to the MLF all along the tract, and S/it1 expressed dorsal to the MLF at the isthmus (Fig. 6 E,F). The broad ventral expression domain of the Robo1 receptor includes the area of the nMLF, indicating that it might constitute the receptor mediating Slit repulsion (Fig. $6 \mathrm{G}, \mathrm{H}$ ). This expression pattern is consistent with Slits being involved in the fasciculation of the MLF, alike their role in the rostrally located tract of the postoptic commissure (Devine and Key, 2008).

Previous studies in Xenopus have already shown that DCC (Anderson et al., 2000a) and Neogenin (Wilson and Key, 2006) play a role in the guidance of early tracts in the forebrain, notably the longitudinally projecting tract of the postoptic commissure (TPOC). Our results indicate that Netrin signalling, in combination with Slits, likewise could be involved in guiding the longitudinal tracts in pretectum and midbrain. Consistent with this hypothesis, a recent study in mouse has implicated Slit-Robo signalling with the correct guidance of the early longitudinal tracts (Farmer et al., 2008).

\section{Expression of guidance molecules at choice points}

In contrast to the extended expression domains associated with the longtitudinal fibre systems, several axon guidance molecules show localised expression domains that are correlated with choice points for specific tracts. For the MLF, the first decision is to project axons caudally, not rostrally. A distinct expression domain of Sema3A was found located rostral and ventral to the nMLF (Fig. 6 I,J), while the nMLF itself expressed Neuropilin1 and PlexinA4 already during initial axon outgrowth (Fig. $6 \mathrm{~K}, \mathrm{~L}$ ). Interestingly, in zebrafish Neuropilin la is expressed within cells lying along the pathways of the PC and the MLF (Yu et al., 2004). However, in zebrafish Sema3D expression lies rostral to the nMLF, and in Sema3Ddeficient embryos, axons of the MLF extend rostrally into 

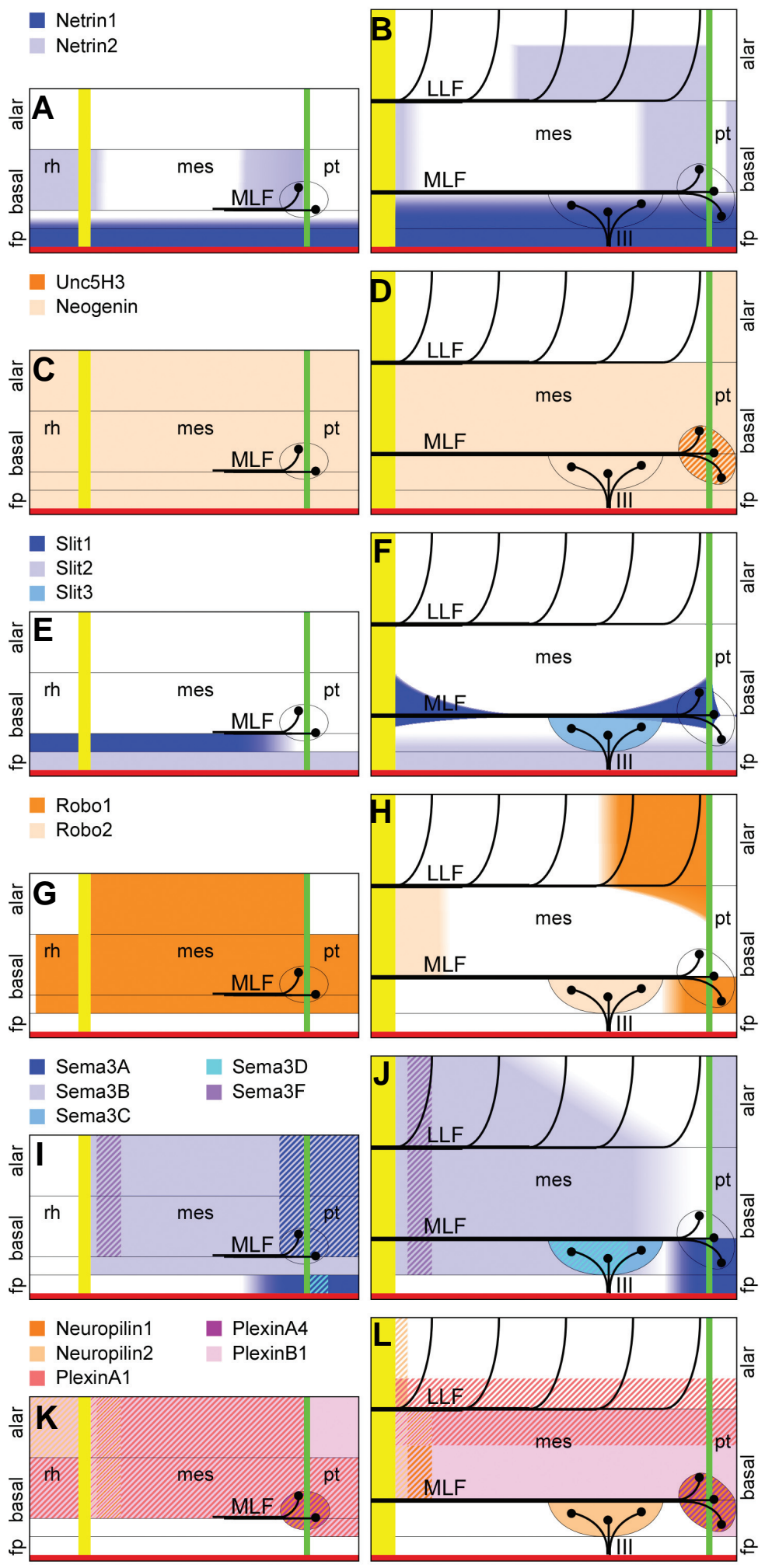

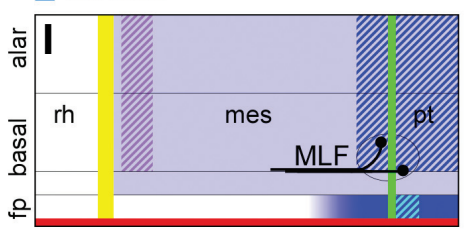

Fig. 6. Schematic representation of axon guidance molecule expression patterns in the mesencephalon. This diagram represents the mesencephalic region analysed at E2 $(\mathbf{A}, \mathbf{C}, \mathbf{E}, \mathbf{G}, \mathbf{I}, \mathbf{K})$ and E3 (B, D, $\mathbf{F}, \mathbf{H}, \mathbf{J}, \mathbf{L})$. The MFB is represented by a vertical green line, the $M H B$ is shown by a vertical yellow line and the midline is shown as a horizontal red line. Thin horizontal lines separate floor plate, basal plate and alar plate, with a further horizontal line marking the course of the MLF in the basal plate, which forms an expression boundary within the basal plate for some of the genes. Neurons are shown as black circles, with axons representing the MLF, LLF and oculomotor nerve depicted as thick black lines. Gene expression domains are shown as coloured areas, hatched pattern indicating co-expression of genes. (A) At E2, Netrin1 (dark blue) expression in the floor plate extends across hindbrain, midbrain and pretectum, dorsally reaching into the basal plate. Netrin2 (light blue) expression is restricted to the basal plate around the MHB and a small area in the rostral mesencephalon. (B) At E3, the Netrin1 expression domain, in addition to the floor plate, includes the ventral basal plate up to the MLF. Netrin2 is still expressed in the basal plate of caudal and rostral mesencephalon. Further Netrin2 expression domains are the rostral alar plate of the mesencephalon and the basal plate of the rostral pretectum. (C) Neogenin (light orange) is expressed throughout the neural tube at E2, while no Unc5H3 (dark orange) signal is observed. (D) At E3, Neogenin expression remains expressed along the mesencephalon, but predominantly in the basal plate. Unc5H3 expression overlaps with the $n M L F$. (E) At E2, Slit1 (dark blue) is expressed in the ventral basal plate, weaker at the rostral end of the mesencephalon and more strongly towards the caudal mesencephalon and hindbrain. Slit2 (light blue) is expressed throughout the entire length of the floor plate. No Slit3 (mid blue) expression is observed at E2. (F) Slit2 expression remains throughout the floor plate and ventral basal plate at E3. Slit1 is expressed in two domains in the mesencephalic basal plate, the first rostrally around the MFB and the $n M L F$, and the second domain caudally. Slit3 expression overlies the oculomotor nucleus. (G) At E2, Robo1 (dark orange) is expressed throughout the basal and alar plates, whilst no Robo2 expression (light orange) is observed. (H) During later stages of development, Robo1 expression becomes restricted to two domains: the first domain is positioned ventral to the $M M L F$ in the basal plate of pretectum and rostral mesencephalon; the second domain is located in the rostral alar plate of the mesencephalon, abutting the MFB. Robo2 is also expressed in two domains, the first overlapping with the oculomotor nucleus and the second in the basal plate of MHB and caudal mesencephalon. (I) At E2, Sema3A (dark blue) is expressed rostral and ventral to the $n M L F$. Sema3B (light blue) is expressed throughout the mesencephalon and pretectum except the floor plate. Sema3C (mid blue) is not yet expressed at E2. Sema3D (turquoise) is expressed in the floor plate of the pretectum. Sema3F (purple) expression forms a stripe in the caudal mesencephalon, just rostral the MHB. (J) At later stages, Sema3A expression remains both rostral and ventral to the $n M L F$. Sema3B expression in the mesencephalon is becoming more restricted to the basal plate. Sema3C, and and later also Sema3D expression overlies with the oculomotor nucleus. Sema3C spans the length of the oculomotor nucleus whilst Sema3D expression is more restricted to the caudal region of the oculomotornucleus. Sema3F expression remains within the caudal mesencephalon. (K) At E2, Neuropilin1 (dark orange) and PlexinA4 (dark magenta) expression domains overlap with the $n M L F$. Neuropilin1 signals also form a stripe in the caudal mesencephalon, similar to Sema3F signals along the MHB. Neuropilin2 (light orange) is expressed in the alar plate of caudal mesencephalon and MHB. PlexinA1 (light red) and PlexinB1 (light magenta) are both expressed broadly in the neural tube, ventrally delimited by the MLF. (L) At E3, there is no change in Neuropilin1 and PlexinA4 expression. PlexinB1 signals become more restricted to the basal plate, while PlexinA1 expression is strongest around the LLF. Neuropilin2 expression is maintained at the MHB, and an additional expression domain is found in the oculomotor nucleus. Key: III (oculomotor nerve), fp (floor plate), LLF (lateral longitudinal fascicle), MLF (medial longitudinal fascicle), mes (mesencephalon), pt (pretectum), rh (rhombencephalon). 
the forebrain (Wolman et al., 2004). In chick, no Sema3Dexpression is observed in this region, but the Sema3A expression pattern is comparable to that of Sema3D in zebrafish, and Sema3A may play a similar role in controlling the outgrowth of MLF axons. At later stages, when the MLF is already well established, Sema3A has been shown to be expressed within the mesencephalon, where it repels the axons of the tectobulbar tract, preventing these axons from crossing over the MLF and directing them in a caudal direction (Henke-Fahle et al., 2001).

In Xenopus, NOC2-positive axons originating in the telencephaIon project via the tract of the postoptic commissure (Anderson and Key, 1999). At the MFB, some of these axons cross the ventral midline to form the ventral commissure, while others join the MLF (Anderson et al., 2000b). Sema3 signalling via Neuropilin1 is implicated in this decision in Xenopus (Anderson etal., 2000b), and interestingly in the chick Sema3D is expressed in the ventral midline at the MFB at early stages (Fig. 6I).

The axons of the PC start to extend out later than the MLF axons, around HH17 (Chedotal et al., 1995). The PC is limited to the caudal portion of the pretectum, where the PC axons initially project dorsally towards the roof plate. Netrin2 is expressed in the ventral mesencephalon and pretectum, but notably excluding the path of the PC in the caudal pretectum (Fig. 6B). The expression of Unc5H3 overlaps the nMLF (Fig. 6D), but the ventrally located neurons projecting into the tract of the $\mathrm{PC}$ are intermingled with the MLF neurons (Schubert and Lumsden, 2005), and thus might also express this receptor. Hence, repulsion by Netrin2 could be involved in the positioning of the PC to the caudal pretectum.

\section{Guidance cues for the cranial nerves}

Our analysis included two of the cranial nerves, the trochlear formed in the isthmus, and the oculomotor developing in the ventral midbrain. The receptors Robo2 and Neuropilin2 are prominently expressed in the oculomotor nucleus (Fig. $6 \mathrm{H}, \mathrm{L}$ ), indicating that oculomotor neurons could be responsive to Slit and Sema3 signalling. At later stages, Slit3is present at the oculomotor and trochlear nuclei (Fig. 6F). Likewise, Sema3C and Sema3D expression are found in the oculomotor nucleus (Fig. 6J), as previously described by Chilton and Guthrie (Chilton and Guthrie, 2003; Chilton and Guthrie, 2004). While Sema3C expression has been observed in the nuclei of all cranial motorneurons (Chilton and Guthrie, 2003; Melendez-Herrera and Varela-Echavarria, 2006) the expression of Sema3D starts later than Sema3C, and is only observed in a subset of oculomotor neurons (Fig. 6J). The restricted expression of Sema3D could be significant with respect to the differential innervation of the eye muscles by specific subnuclei in the oculomotor complex (Chilton and Guthrie, 2004; Heaton and Wayne, 1983). In rat, Sema3Cis additonally expressed within the pretectum and controls the outgrowth of axons of dopaminergic neurons (Hernandez-Montiel et al., 2008). As previously described (Watanabe et al., 2004), Sema3F expression aligns the rostral border of the isthmus (Fig. $6 \mathrm{I}, \mathrm{J}$ ), where it has been shown to play a role in guiding trochlear axons along the MHB (Watanabe et al., 2004).

\section{Conclusions}

By analysing the expression patterns of axon guidance molecules within mesencephalon and pretectum during early stages of chick brain development, we have shown that a number of guidance cues are present during the initial formation of the early axon scaffold. Some of the guidance molecules like Netrins and Slits could be involved in establishing the longitudinal tracts, while others like Class3 Semaphorins are more likely to guide specific axons at choice points.

\section{Materials and Methods}

Fertilised chicken eggs were obtained from Henry Stewart Ltd. and incubated at $38^{\circ} \mathrm{C}$ for 2, 3 or 4 days. Embryos were harvested in PBS, fixed in $4 \%$ paraformaldehyde, and staged according to Hamburger \& Hamilton (1951).

In situ hybridisation and immunohistochemistry followed previously described procedures (Schubert and Lumsden, 2005). Briefly, following bleaching and detergent treatment the embryos were prehybridised in hybridisation buffer ( $50 \%$ formamide/5x SSC/2\% SDS/2\% Boehringer blocking reagent $/ 250 \mu \mathrm{g}^{*} \mathrm{ml}^{-1} \mathrm{RNA} / 100 \mathrm{gg}^{*} \mathrm{ml}^{-1}$ heparin), before adding the Digoxigenin-labelled probe. After hybridisation, the embryos were washed in $50 \%$ formamide/2x SSC $/ 1 \%$ SDS, before being transferred to maleic acid buffer. They were incubated with the alkaline phosphataseconjugated anti-Digoxigenin antibody (Roche), and finally the signal was detected using NBT/BCIP (Roche) as substrate. Embryos were then refixed in $4 \%$ paraformaldehyde before proceeding to the immunohistochemistry. They were incubated in anti-Neurofilament antibody (Zymed RMO227, used 1:1000), followed by the secondary antibody (Jackson Laboratories Peroxidase-conjugated goat anti-mouse, used 1:100), finally detected with Diaminobenzidine (Vector Labs HRP kit) as substrate.

Probes used for the in situ hybridisations were either provided by colleagues or were EST clones obtained from MRC Geneservice. Probes for Netrin1 and Netrin2(Serafini etal., 1994) were kind gifts by M. TessierLavigne, while for Neogenin (chEST741p10) and Unc5H3(chEST1023g17) we used EST clones. Slit1 and Slit2were gifts from A. Chédotal, Slit3was a gift from L. Erskine and N. Vargesson, and Robo1and Robo2(Vargesson et al., 2001) were gifts from E. Laufer. Sema3C, Sema3D, and Sema3E (Feiner et al., 1997; Luo et al., 1995) were gifts from J. Raper. Sema3A (chEST712j10), Sema3B (chEST771a21), and Sema3F(ROSO12B12) were EST's. Neuropilin1 (Takagi et al., 1995) was a gift from H. Fujisawa, and Neuropilin2 (Watanabe et al., 2004) was a gift from Y. Watanabe. Probes for PlexinA1 (chEST21d16), PlexinA4 (chEST 744O11), and PlexinB1 (chEST1023G17) were all derived from EST's.

\section{Acknowledgements}

We would like to thank A. Chédotal, L. Erskine, H. Fujisawa, E. Laufer J. Raper, M. Tessier-Lavigne, N. Vargesson and Y. Watanabe for kindly sharing probes. We would also like to thank $F$. Winterbottom and $S$. Dietrich for their help in ironing out problems with the in situ hybridisation. The project was supported by the Royal Society and by the European Union (Interreg IV AdMiN project), and our research on the early axon scaffold has been funded by a project grant from the BBSRC (BB) C510332). S. G. was supported by an Undergraduate Research Bursary from the Nuffield Foundation.

\section{References}

AHSAN, M., RILEY, K.L. and SCHUBERT, F.R. (2007). Molecular mechanisms in the formation of the medial longitudinal fascicle. J Anat 211: 177-187.

ANDERSON, R.B., COOPER, H.M., JACKSON, S.C., SEAMAN, C. and KEY, B. (2000a). DCC plays a role in navigation of forebrain axons across the ventral midbrain commissure in embryonic Xenopus. Dev Bio/217: 244-253.

ANDERSON, R.B., JACKSON, S.C., FUJISAWA, H. and KEY, B. (2000b). Expres sion and putative role of neuropilin-1 in the early scaffold of axon tracts in embryonic Xenopus brain. Dev Dyn 219: 102-128. 
ANDERSON, R.B. and KEY, B. (1999). Novel guidance cues during neuronal pathfinding in the early scaffold of axon tracts in the rostral brain. Development 126: $1859-1868$.

ANDREWS, G.L., TANGLAO, S., FARMER, W.T., MORIN, S., BROTMAN, S., BERBEROGLU, M.A., PRICE, H., FERNANDEZ, G.C., MASTICK, G.S., CHARRON, F. et al. (2008). Dscam guides embryonic axons by Netrindependent and -independent functions. Development 135: 3839-3848.

BROSE, K., BLAND, K.S., WANG, K.H., ARNOTT, D., HENZEL, W., GOODMAN, C.S., TESSIER-LAVIGNE, M. and KIDD, T. (1999). Slit proteins bind Robo receptors and have an evolutionarily conserved role in repulsive axon guidance. Cel/96: 795-806.

CHEDOTAL, A., POURQUIE, O. and SOTELO, C. (1995). Initial tract formation in the brain of the chick embryo: selective expression of the BEN/SC1/DM-GRASP cell adhesion molecule. Eur JNeurosci7: 198-212.

CHILTON, J.K. (2006). Molecular mechanisms of axon guidance. Dev Bio/292: 1324.

CHILTON, J.K. and GUTHRIE, S. (2003). Cranial expression of class 3 secreted semaphorins and their neuropilin receptors. Dev Dyn 228: 726-733.

CHILTON, J.K. and GUTHRIE, S. (2004). Development of oculomotor axon projections in the chick embryo. J Comp Neuro/472: 308-317.

CHITNIS, A.B. and KUWADA, J.Y. (1990). Axonogenesis in the brain of zebrafish embryos. JNeurosci10: 1892-1905.

CHITNIS, A.B. and KUWADA, J.Y. (1991). Elimination of a brain tract increases errors in pathfinding by follower growth cones in the zebrafish embryo. Neuron 7: $277-285$

COOPER, H.M. (2002). Axon guidance receptors direct growth cone pathfinding: rivalry at the leading edge. Int $J$ Dev Bio/46: 621-631.

DEVINE, C.A. and KEY, B. (2008). Robo-Slit interactions regulate longitudinal axon pathfinding in the embryonic vertebrate brain. Dev Bio/313: 371-383.

DICKSON, B.J. (2002). Molecular mechanisms of axon guidance. Science 298: 1959-64.

DICKSON, B.J. and GILESTRO, G.F. (2006). Regulation of commissural axon pathfinding by slit and its Robo receptors. Annu Rev Ce/l Dev Bio/22: 651-675.

DOLDAN, M.J., PREGO, B., HOLMQVIST, B., HELVIK, J.V. and DE MIGUEL, E. (2000). Emergence of axonal tracts in the developing brain of the turbot (Psetta maxima). Brain Behav Evo/56: 300-309.

EASTER, S.S., JR., ROSS, L.S. and FRANKFURTER, A. (1993). Initial tract formation in the mouse brain. JNeurosci13: 285-299.

FARMER, W.T., ALTICK, A.L., NURAL, H.F., DUGAN, J.P., KIDD, T., CHARRON, F. and MASTICK, G.S. (2008). Pioneer longitudinal axons navigate using floor plate and Slit/Robo signals. Development 135: 3643-3653.

FAZELI, A., DICKINSON, S.L., HERMISTON, M.L., TIGHE, R.V., STEEN, R.G., SMALL, C.G., STOECKLI, E.T., KEINO-MASU, K., MASU, M., RAYBURN, H. et al. (1997). Phenotype of mice lacking functional Deleted in colorectal cancer (Dcc) gene. Nature 386: 796-804.

FEINER, L., KOPPEL, A.M., KOBAYASHI, H. and RAPER, J.A. (1997). Secreted chick semaphorins bind recombinant neuropilin with similar affinities but bind different subsets of neurons in situ. Neuron 19: 539-545.

GAD, J.M., KEELING, S.L., WILKS, A.F., TAN, S.S. and COOPER, H.M. (1997). The expression patterns of guidance receptors, DCC and Neogenin, are spatially and temporally distinct throughout mouse embryogenesis. Dev Biol 192: $258-273$.

HAMBURGER, V. and HAMILTON, H.L. (1951). A series of normal stages in the development of the chick embryo. J. Morphol. 88: 49-92.

HARTENSTEIN, V. (1993). Early pattern of neuronal differentiation in the Xenopus embryonic brainstem and spinal cord. J Comp Neuro/328: 213-231.

HE, Z. and TESSIER-LAVIGNE, M. (1997). Neuropilin is a receptor for the axonal chemorepellent Semaphorin III. Cel/90: 739-751.

HEATON, M.B. and WAYNE, D.B. (1983). Patterns of extraocular innervation by the oculomotor complex in the chick. J Comp Neuro/216: 245-252.

HENKE-FAHLE, S., BECK, K.W. and PUSCHEL, A.W. (2001). Differential responsiveness to the chemorepellent Semaphorin $3 A$ distinguishes Ipsi- and contralaterally projecting axons in the chick midbrain. Dev Bio/237: 381-397.

HERNANDEZ-MONTIEL, H.L., MELENDEZ-HERRERA, E., CEPEDA-NIETO, A.C., MEJIA-VIGGIANO, C., LARRIVA-SAHD, J., GUTHRIE, S. and VARELA-
ECHAVARRIA, A. (2003). Diffusible signals and fasciculated growth in reticulospinal axon pathfinding in the hindbrain. Dev Bio/255: 99-112.

HERNANDEZ-MONTIEL, H.L., TAMARIZ, E., SANDOVAL-MINERO, M.T. and VARELA-ECHAVARRIA, A. (2008). Semaphorins 3A, 3C, and 3F in mesencephalic dopaminergic axon pathfinding. J Comp Neuro/506: 387-397.

HJORTH, J. and KEY, B. (2002). Development of axon pathways in the zebrafish central nervous system. Int J Dev Bio/46: 609-619.

HJORTH, J.T. and KEY, B. (2001). Are pioneer axons guided by regulatory gene expression domains in the zebrafish forebrain? High-resolution analysis of the patterning of the zebrafish brain during axon tract formation. Dev Bio/229: 271 286.

ISHIKAWA, Y., KAGE, T., YAMAMOTO, N., YOSHIMOTO, M., YASUDA, T., MATSUMOTO, A., MARUYAMA, K. and ITO, H. (2004). Axonogenesis in the medaka embryonic brain. J Comp Neuro/476: 240-253.

KEINO-MASU, K., MASU, M., HINCK, L., LEONARDO, E.D., CHAN, S.S., CULOTTI J.G. and TESSIER-LAVIGNE, M. (1996). Deleted in Colorectal Cancer (DCC) encodes a netrin receptor. Ce//87: 175-185.

KENNEDY, T.E., SERAFINI, T., DE LA TORRE, J.R. and TESSIER-LAVIGNE, M. (1994). Netrins are diffusible chemotropic factors for commissural axons in the embryonic spinal cord. Cel/78: 425-435.

KOLODKIN, A.L., LEVENGOOD, D.V., ROWE, E.G., TAI, Y.T., GIGER, R.J. and GINTY, D.D. (1997). Neuropilin is a semaphorin III receptor. Cel/90: 753-762.

LEONARDO, E.D., HINCK, L., MASU, M., KEINO-MASU, K., ACKERMAN, S.L. and TESSIER-LAVIGNE, M. (1997). Vertebrate homologues of C. elegans UNC-5 are candidate netrin receptors. Nature 386: 833-838.

LUO, Y., RAIBLE, D. and RAPER, J.A. (1993). Collapsin: a protein in brain that induces the collapse and paralysis of neuronal growth cones. Ce//75:217-227.

LUO, Y., SHEPHERD, I., LI, J., RENZI, M.J., CHANG, S. and RAPER, J.A. (1995) A family of molecules related to collapsin in the embryonic chick nervous system. Neuron 14: 1131-1140.

MASTICK, G.S. and EASTER, S.S., JR. (1996). Initial organization of neurons and tracts in the embryonic mouse fore- and midbrain. Dev Bio/173: 79-94.

MELENDEZ-HERRERA, E. and VARELA-ECHAVARRIA, A. (2006). Expression of secreted semaphorins and their receptors in specific neuromeres, boundaries, and neuronal groups in the developing mouse and chick brain. Brain Res 1067 126-137.

MOLLE, K.D., CHEDOTAL, A., RAO, Y., LUMSDEN, A. and WIZENMANN, A. (2004). Local inhibition guides the trajectory of early longitudinal tracts in the developing chick brain. Mech Dev 121: 143-156.

MOORE, S.W., TESSIER-LAVIGNE, M. and KENNEDY, T.E. (2007). Netrins and their receptors. Adv Exp Med Bio/621: 17-31.

NIEUWENHUYS, R. (1998). Development of fibre systems. In The central nervous system of vertebrates, vol. 1 (ed. NIEUWENHUYS, R.TEN DONKELAAR, H. J. and NICHOLSON, C.). Springer-Verlag, Berlin Heidelberg, pp. 256-271.

PATEL, C.K., RODRIGUEZ, L.C. and KUWADA, J.Y. (1994). Axonal outgrowth within the abnormal scaffold of brain tracts in a zebrafish mutant. JNeurobio/25: 345-360.

RAPER, J.A. (2000). Semaphorins and their receptors in vertebrates and invertebrates. Curr Opin Neurobio/10: 88-94.

SCHUBERT, F.R. and LUMSDEN, A. (2005). Transcriptional control of early tract formation in the embryonic chick midbrain. Development 132: 1785-1793.

SERAFINI, T., KENNEDY, T.E., GALKO, M.J., MIRZAYAN, C., JESSELL, T.M. and TESSIER-LAVIGNE, M. (1994). The netrins define a family of axon outgrowthpromoting proteins homologous to C. elegans UNC-6. Cel/78: 409-424.

TAKAGI, S., KASUYA, Y., SHIMIZU, M., MATSUURA, T., TSUBOI, M., KAWAKAMI, A. and FUJISAWA, H. (1995). Expression of a cell adhesion molecule, neuropilin, in the developing chick nervous system. Dev Bio/170: 207-222.

TAKAHASHI, T., FOURNIER, A., NAKAMURA, F., WANG, L.H., MURAKAMI, Y. KALB, R.G., FUJISAWA, H. and STRITTMATTER, S.M. (1999). Plexinneuropilin-1 complexes form functional semaphorin-3A receptors. Ce//99: 59 69.

TAMAGNONE, L., ARTIGIANI, S., CHEN, H., HE, Z., MING, G.I., SONG, H. CHEDOTAL, A., WINBERG, M.L., GOODMAN, C.S., POO, M. et al. (1999). Plexins are a large family of receptors for transmembrane, secreted, and GPIanchored semaphorins in vertebrates. Cel/99: 71-80. 
TEAR, G., SEEGER, M. and GOODMAN, C.S. (1993). To cross or not to cross: a genetic analysis of guidance at the midline. Perspect Dev Neurobio/1: 183-194.

TESSIER-LAVIGNE, M. and GOODMAN, C.S. (1996). The molecular biology of axon guidance. Science 274: 1123-1133.

VARGESSON, N., LURIA, V., MESSINA, I., ERSKINE, L. and LAUFER, E. (2001). Expression patterns of Slit and Robo family members during vertebrate limb development. Mech Dev 106: 175-180.

VIELMETTER, J., KAYYEM, J.F., ROMAN, J.M. and DREYER, W.J. (1994). Neogenin, an avian cell surface protein expressed during terminal neuronal differentiation, is closely related to the human tumor suppressor molecule deleted in colorectal cancer. J Cel/ Bio/127: 2009-2020.

WATANABE, Y., TOYODA, R. and NAKAMURA, H. (2004). Navigation of trochlear motor axons along the midbrain-hindbrain boundary by neuropilin 2. Development 131: 681-692.

WILSON, N.H. and KEY, B. (2006). Neogenin interacts with RGMa and netrin-1 to guide axons within the embryonic vertebrate forebrain. Dev Bio/296: 485-498.

WILSON, S.W., ROSS, L.S., PARRETT, T. and EASTER, S.S., JR. (1990). The development of a simple scaffold of axon tracts in the brain of the embryonic zebrafish, Brachydanio rerio. Development 108: 121-145.

WOLMAN, M.A., LIU, Y., TAWARAYAMA, H., SHOJI, W. and HALLORAN, M.C (2004). Repulsion and attraction of axons by semaphorin3D are mediated by different neuropilins in vivo. J Neurosci24: 8428-8435.

YU, H.H., HOUART, C. and MOENS, C.B. (2004). Cloning and embryonic expres sion of zebrafish neuropilin genes. Gene Expr Patterns 4: 371-378.

\section{Further Related Reading, published previously in the Int. J. Dev. Biol.}

See Special Issue Pattern Formation edited by Michael K. Richardson and Cheng-Ming Chuong at:

http://www.ijdb.ehu.es/web/contents.php?vol=53\&issue=5-6

Axon guidance in the inner ear

Donna M. Fekete and Andrea M. Campero

Int. J. Dev. Biol. (2007) 51: 549-556

Analysis of Netrin 1 receptors during inner ear development

Tanja Matilainen, Maarja Haugas, Jordan A. Kreidberg and Marjo Salminen

Int. J. Dev. Biol. (2007) 51: 409-414

Systematic screening for genes specifically expressed in the anterior neuroectoderm during early Xenopus development

Noriyuki Takahashi, Naoko Tochimoto, Shin-Ya Ohmori, Hiroshi Mamada, Mari Itoh, Masako Inamori, Jun Shinga, Shin-Ichi Osada and Masanori Taira

Int. J. Dev. Biol. (2005) 49: 939-951

New views on retinal axon development: a navigation guide

Fanny Mann, William A. Harris and Christine E. Holt

Int. J. Dev. Biol. (2004) 48: 957-964

Development of axon pathways in the zebrafish central nervous system Jensen Hjorth and Brian Key

Int. J. Dev. Biol. (2002) 46: 609-619

Axon guidance receptors direct growth cone pathfinding: rivalry at the leading edge

Helen M Cooper

Int. J. Dev. Biol. (2002) 46: 621-631

The isthmic organizer and brain regionalization

S Martínez

Int. J. Dev. Biol. (2001) 45: 367-371

5 yr ISI Impact Factor $(2008)=3.271$

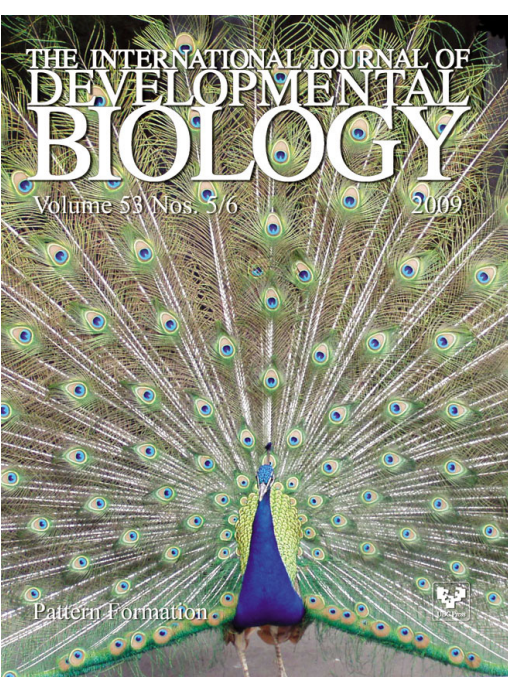

